\title{
Characterization of Polymer Materials by Fluorescence Imaging
}

\author{
W. Heckmann
}

\author{
BASF AG, Polymer Physics Department, D 67056 Ludwigshafen, Germany
}

Characterization of polymer materials by fluorescence imaging offers valuable additional information to transmission electron microscopy. Only a few polymers show an autofluorescence effect for visible light excitation. The polyurethanes and poly(phenylene ether) belong to these groups and can be studied in fluorescence without staining. The majority of polymers however have to be stained with fluorophores to be imaged. Neocarmine, Lumogen F-Rot 300, Fluoresceine and Rhodamine, are important dyes for staining polymers. Some examples on Neocarmine- and Lumogen-staining are explained below. All images were obtained with a Leica TCS4D Laser Scan Microscope (CLSM).

A) Staining of Polyamide with Neocarmine [1] In a 90:10 Polypropylene(PP)/Polyamid(PA)-blend the cut surface was stained with Neocarmine, a fluorescence dye, which interacts only with the PA component. Excitation was achieved by irradiation with green light $(568 \mathrm{~nm})$ and on the emission side a longwave pass filter (LP 590) was used. Fig. 1a shows fluorescing polyamide particles in green and the PP-matrix in black. Another application for the Neocarmine fluorescence stain are blends of glass fiber reinforced Polyethersulfone (PES) and Polyamide (PA). Small pieces of injection-moulded pellets were ground and polished before staining with Neocarmin. In the image of Fig. 1b the PES-phase and the glass fibers are imaged green and the PA-phase is imaged in red. A cocontinuous network structure is observed with an enrichment of PA around the glass fibers.

B) Staining of Oil-droplets with Lumogen red 300 [2]

To study the diffusion of a hand cream into paint films a hand cream was formulated where the oil droplets were stained with Lumogen red 300, an oil soluble fluorescence dye (Fig. 2a). The hand cream was applied on the surface of dispersion films for different lengths of time. The diffusion of the oil phase of the hand creme can then be followed by fluorescence imaging of the cross sections. In Fig. $2 b$ such a series of images are shown.

C) Autofluorescence

Polyurethanes are one of the few polymers showing autofluorescence on excitation with visible light $(488 \mathrm{~nm})$. In rigid foams besides the struts also the foam lamellae can be imaged, especially in the normal reflection mode. Due to interference effects, lines of equal thickness are imaged. In a combination image (reflection/fluorescence) the complete foam cell can be visualized (Fig. 3a). Cross sections of PA-fibers often show a thin fluorescent surface layer (Fig. 3b). As PA exhibits autofluorescence if it is treated at higher temperature under air we believe that the thin surface layer is due to thermal oxidation. Pigments within the fiber cross section are imaged by the normal reflection mode.

References

[1] G. Illing, Angew. Makromol. Chem. 95(1981)83

[2] G. Seybold and G. Wagenblast, Dyes and Pigments 11(1989)303 


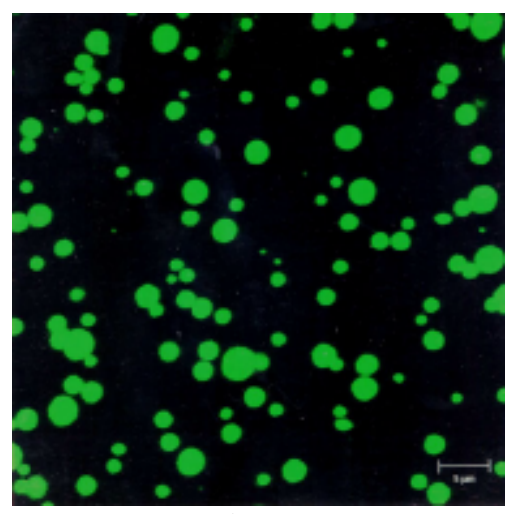

a)

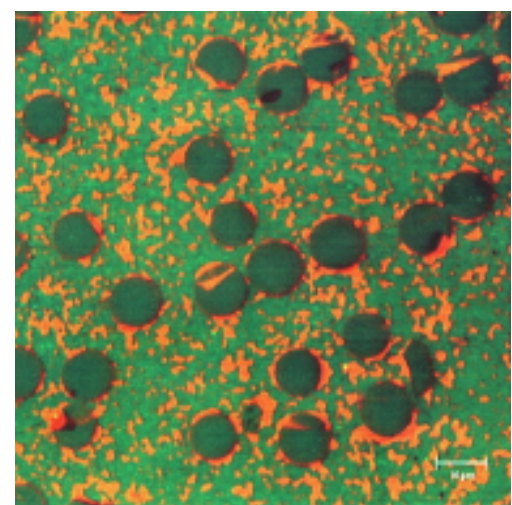

b)

Fig. 1: CLSM fluorescence images of a) a cut face of a PP/PA-Blend and b) a ground and polished pellet of a PES/PA/GF-blend after staining with Neocamine

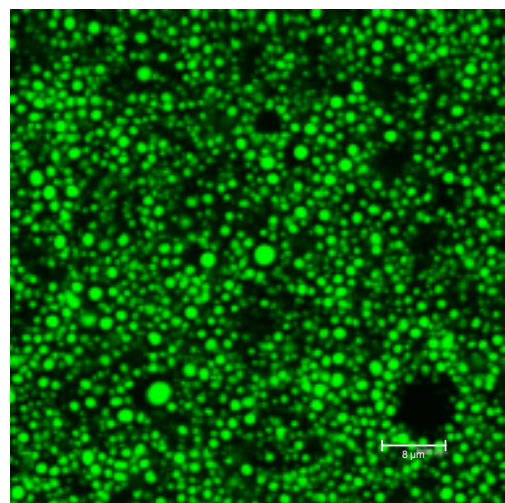

a)

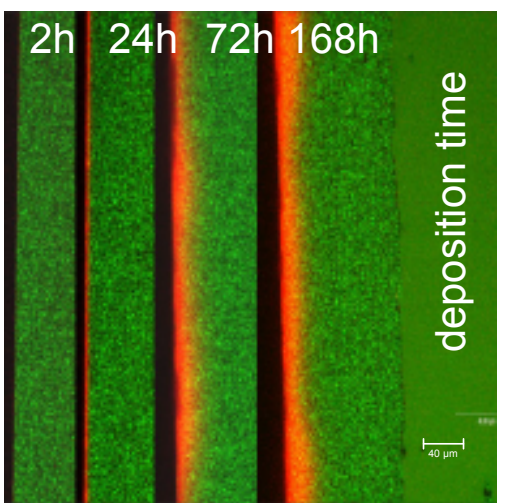

b)

Fig. 2: Fluorescence images of a) a thin film of a hand cream, oil droplets stained with Lumogen red $300 \mathrm{~b}$ ) kinetics of hand cream penetration into an acrylic dispersion film. Excitation wavelength $568 \mathrm{~nm}$.

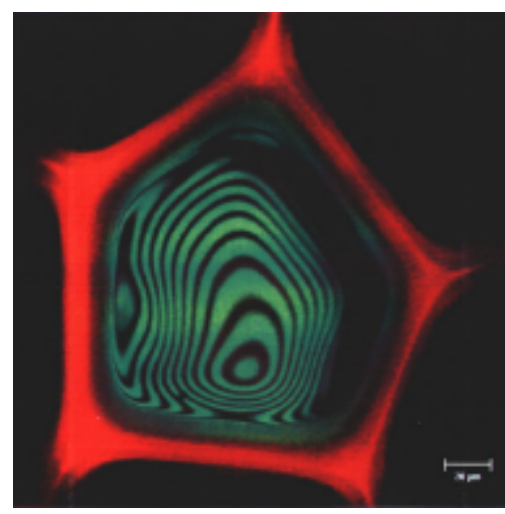

a)

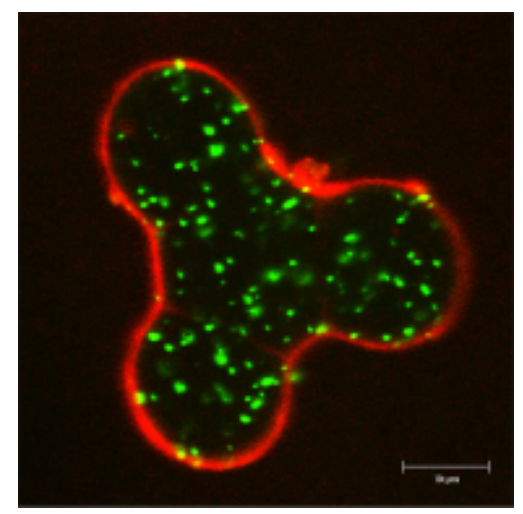

b)

Fig. 3: a) composite image (reflection/fluorescence) of a rigid PUR-foam cell b) optical cross section through a trilobal fiber, pigments within the fiber cross section are shown in green. 